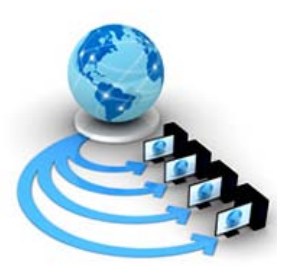

Volume 8, No. 8, September-October 2017

International Journal of Advanced Research in Computer Science

RESEARCH PAPER

Available Online at www.ijarcs.info

\title{
FAULT-DETECTION ON MULTI-ROBOT PATH PLANNING
}

\author{
Ashutosh Kumar Singh \\ Department of Computer Science \& Engineering \\ Motilal Nehru National Institute of Technology Allahabad \\ Allahabad, India
}

\author{
Naveen Kumar \\ Department of Computer Science \& Engineering \\ Motilal Nehru National Institute of Technology Allahabad \\ Allahabad, India
}

\begin{abstract}
This One of the essential issues in coordinated multi-robot path planning and area exploration is how to allocate target location to the individual robot and how to superior share out the robots in the environment. In this process, they must have an optimal motion planning algorithm to find out the minimum path with the lesser amount of time and minimum amount of energy. Given environment is divided into subarea equal to the available number of robots using circle partitioning method. Every robot finds the shortest path for the given subarea assigned to them. It may be possible that in any sector there is a high density of obstacles or path planning capability of any robot is not good as compared to others. So path planning in that sector or by that robot will be done easily. Our involvement is in three fold: (1) EA* algorithm for path planning; (2) assignment technique is to better share out the robots in the environment; (3) fault-detection algorithm, if one or more robot may fail during the path planning. Our proposed algorithm has been tested for the different environments with the different level of complexity depending on the number of obstacles. Here, the fault-detection concept is added that means if any robot may fail during the path planning then it can be handled easily.
\end{abstract}

Keywords: Multi-Robot; Path Planning; A* Algorithm; Energy Consumption; Fault-Detection; Area Exploration

\section{INTRODUCTION}

Each robotic and intelligent system uses planning to decide the motion of the robot in the real word environment. Robot Path Planning is a fundamental problem in the field of robotics that tries to find and optimize the shortest path from the initial position (source) to target position (destination). There are several paths available to achieve this task, but in fact, one of the best paths is selected according to few guidelines. These guidelines are the shortest path (from the initial position to target position), least energy consuming and shortest time [1, 2]. When we are doing robot area exploration, we are doing robot path planning in such a way that if we divide the whole area in the grid the robot visits every block of the grid at least once. When we are talking about the multiple robots, it is not necessary that each robot visits every block, but when we add all the blocks visited by each robot, then we get the whole area explored. Efficient path planning and exploration of unidentified environments is the key problem in robotics. Like Path planning, area exploration and map construction become more and more robust on the single robot; the subsequently level challenge is to expand these techniques to groups of bots that mean increase the number of robots. Evaluated to the troubles happening in a single robot map coverage and path planning the expansion to more number of robots causes a number of new challenges, i) Synchronization between robots; ii) integration of knowledge composed by the individual robot into the reliable map; and iii) handle with the restricted communication.

In this paper, we have proposed a new approach for multirobot path planning for the unknown environment and this method is based on the circle partitioning concept. The remaining paper is structured as follows: Section 2 explains the related work. Section 3 presents the problem formulation. Section 4 presents the methodology for Area exploration.
Section 5 shows the simulation result using $\mathrm{C}++$. Paper is concluded in section 6 .

\section{RELATED WORK}

In the study, Naom et al. [1] used a polynomial time redundant coverage algorithm to explore the areas in the minimum amount of time by using multiple robots. The study divides the area into square blocks that are visited by different robots. The study points out that the non-redundant type of methods wastes a lot of resources as well as time in avoiding the exploration of the same area again. Puig et al. [2] used a kMeans based multi-robot exploration planner, in order to ensure a balanced exploration technique where robots do not favor a particular area of exploration by forcing the explicit distribution of robots over the whole area. Both the techniques use different methods to divide the area of exploration, but both of them divide the area in form of square grid.

Travel Cost of robots is minimized in the algorithm used by Sariel et al. [3]. The study found that MSF solution is inefficient as there is no calculation of traveling cost of jumping from one branch to another while doing the heuristics of route construction for MST. So, an efficient method that determines how the robots will travel across the targets was proposed using open loop TSP heuristic cost functions. From all unallocated targets, each robot chooses targets from the set of all unallocated targets with preference to the targets closest to it than to its counterparts. Then each robot finds and selects a target with the lowest cost according to a heuristic function from the set of available unallocated targets. The problem with this method is that the bots lose a lot of time in case of conflict decisions and in some cases some bots are at idle state as other bots take the nearby targets.

Senthil et al. [4] suggested another way of reducing the redundancy, and they have divided the whole area into the grids. The spanning Tree Coverage (ES-MSTC*) method is 
used for traversing the blocks and made it linear, so there is no or minimal conflict as the robots travel in parallel straight lines. But authors did not consider an irregular obstacle. Moreover, the robots were supposed ant-sized, so there is a huge problem in actual physical implementation at such a scale.

Communication among the robots while using a multi-robot exploration task is a major issue. Sheng et al. [5] in their study made a very realistic remark that when robots are working in real environments, there is one factor that significantly changes. This factor is communication between the robots when they deal with a simulated environment, the robots are considered to be in excellent communication, and there is no or negligible environmental effect on the robots while communicating with each other. The scenario completely changes on actual implementation when there is interference of environment and the robots have an insufficient and weak range and signal strength. The author used a distributed bidding algorithm to solve the issue. Chen et al.[6] suggested that the latest wireless ad-hoc networks are a boon to these mobile robots, as they can establish an ad-hoc LAN among them and communicate with each other.

Sheng et al. [7] suggested an algorithm to take care of the communication issues; the research indicated that while allocating areas to be explored it should be kept under consideration that at all times all the robots are communicating and exchanging the exploration data at all times. Garcia et at. [15] discussed the coordinated area exploration by using unsupervised clustering algorithm. Wu et. al [16] presented the idea behind the area exploration through global optimization method. Another research work [19] provides PSO based area exploration for the multi-robots.

The issue of irregular objects is also very important, and the robots need to understand the shape of the objects to avoid them efficiently, irregularly shaped objects cannot be avoided efficiently using linear path propagation methods. Peng et al. [8] in their study used DRS* method to avoid the irregular objects effectively. The method can be compared to robots having sonar to actually determine the obstacles from a distance and start planning an alternate path earlier and also having an earlier path change rather than changes after the collision from the obstacles. The method is very efficient as all the nearby cells can be explored without even visiting them.

Regele et al. [9] suggested another novel approach towards multi-robot area exploration problem. They have suggested rather than leaving the robots in an unplanned fashion, preplan the path and somehow inform synchronization among the robots. The synchronization can be made by doing two things. First one is dividing the area among the robots and the second one, suggestions to remove selfishness from the robots so that they respect the higher priority fags of other robots. By implementing these two things, we can optimize the area explored while minimizing the redundancy. In another study by Low et al. [10] and they point out some hotspots and assign the area to each robot that starts its exploration from that particular hotspot to another fixed destination point. The method is very effective for cases with lower complexity as there is no provision to resolve the conflicts between the robots.
Buggard et al. [11] discuss one common approach developing recently in the area exploration prospect. Their research finds it easier and more optimized if the entire area divided into smaller areas. These areas are assigned to each robot, and there is a minimum redundancy. Another advantage of the technique is the robots require very little time to visit the same area when they work concurrently and without conflicts.

An additional common approach developing recently in the multi-robot area exploration is discussed by Pal et al. $[12,13,14]$. They present multi-robot area exploration for the wireless environment. Here, robot team maintains connection themselves, in such a manner to have an accurate map of the given environment at every moment and having a wellorganized map-reading plan for moving towards the uncovered area. Here author discussed two kinds of troubles concerning to multi-robot area exploration task. First, associated with path planning in which how robot make a plan for their path which takes less amount of energy. Second, the problem is related to preserving the network connectivity in which how they can talking from one to other in such a way to construct global map at each step by exchanging the message with less number of hop count, so that delay in message transmission is minimized.

\section{PROBLEM FORMULATION}

After doing literature survey, we found that the most of the research work is only concentrate on multi-robot area path planning and multi-robot area exploration, but they do not concentrate when some of the robots may fail during the path planning and area exploration. There is another problem with the task assigned to a robot who newly joined the team in the middle of the execution. In which there are some fixed obstacles of random shape and size on the environment. When we are doing the path planning and area exploration, every cell of the network having any one of these three situations: free, filled and unidentified.

- Free represents that there is no obstacle presents in given cell.

- Filled represents that the cell is engaged by obstacles and

- Unidentified represents that the residence of the cell is not recognized until now.

The main aim of the robot to traverse the map at least once. For simplicity, Robot is considered as in the form of the square in such way that the dimension of the robot is equal to the size of the grid cell. With the help of sensor, robot identifies the situation of the grid cell.

\section{Methodology}

Our proposed approach primarily manages environment dividing to finish the investigation as early as possible without any failure. Initially, the area is partitioned into logical subareas utilizing circle dividing technique then robots are assigning to these logical subareas and they begin the investigation inside their logical subareas. To investigate the area robots need to move towards the frontier cell $[17,18]$. 


\section{A. Environment and Robot Region Assignment}

Environment is partitioned in to equal number of sectors. And further divide the whole area in the logical co-centric circles. Because of division higher degree of distribution is attained among the robots. An approach for environment dividing is taking in to account i.e. based on the scheme of circle division. Considering center of the region as the center of the circle, we form a circle that covers the entire area and then divide this circle into number of parts as available robots. By using the concept of circle division the area is divided about equal number of parts. Robots are assigned to each subarea.

\section{B. Implementation of proposed algorithm}

We have implemented the algorithm for the fault-detection on multi-robot path planning. When, we are doing path planning and area exploration by using multiple robots, at that situation some of the robots may fail. This is the problem, and this problem can be removed by using our proposed algorithm. The given environment is divided the number of sectors, and further, it is split into the co-centric circles (tracks). Here we are using EA* algorithm for path planning.

\section{ALGORITHM}

1. MRn: Represent total number of Mobile Robots in the environment

2.Tr: Represent total number of co-centric circles(tracks) in the environment

3. For each Mobile Robot MRi in MRn

4. Do set initial position of each MRi

5. Target covered $=0$, stop $=0$;

6. Total Target covered $=\mathrm{MRn}$;

7. While ( Target covered $<=$ Total Target covered)

8. \{

9. For each(Mobile Robot MRi in MRn)

10. \{

11. if(Mobile Robot MRi.New position is on track surface || Mobile Robot MRi.stop = =True)

$12 .\{$

13. Then for each MRj in (MRn MRi) sends message to

all mobile robots about new updated position

14. If(Mobile Robot MRi.stop = =True)

15.

16.

17.

18.

19.

20.

21.

MRj.Last position

22.

23.

24.

25. \}$$
\}
$$$$
\text { Stop++; }
$$$$
\text { If(MRi.New position = = goal })
$$$$
\{
$$$$
\text { Target covered++; }
$$$$
\text { If(stop }>0)
$$$$
\text { Then Set MRi.New goal }==\text { Stopped }
$$

$$
\}
$$$$
\text { Stop - - ; }
$$$$
\text { 西 }
$$

\section{SIMULATION RESULTS}

In this section, we discuss the Simulation results. Simulations are performed in a workspace with the size of $24 \mathrm{mX} 24 \mathrm{~m}$, in which there exist some circular, square and rectangular (assumption) obstacles with different length and radii. Four robots are coordinated for target based path planning of the environment. The maximum linear velocity of each robot is $0.4 \mathrm{~m} / \mathrm{s}$. Here EA* algorithm is applied to reach to the target avoiding. We have used $\mathrm{C}++$ language to write code for the player server which would be called by simulation for effect. We have considered some parameters for the simulation as given in Table 1.

We have taken two different environments, the first environment having less number of obstacles and the second environment having more number of obstacles

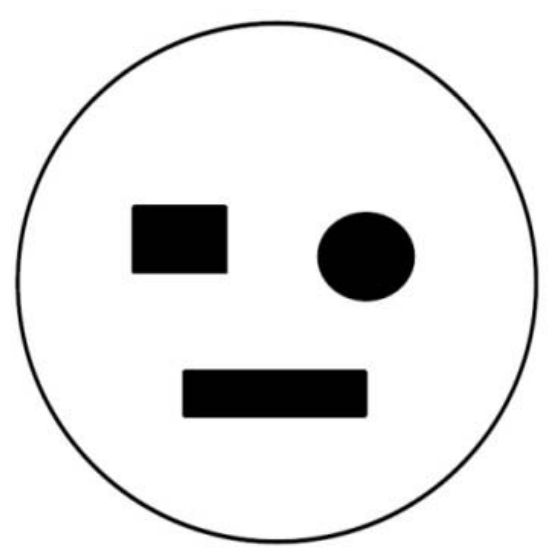

(a)

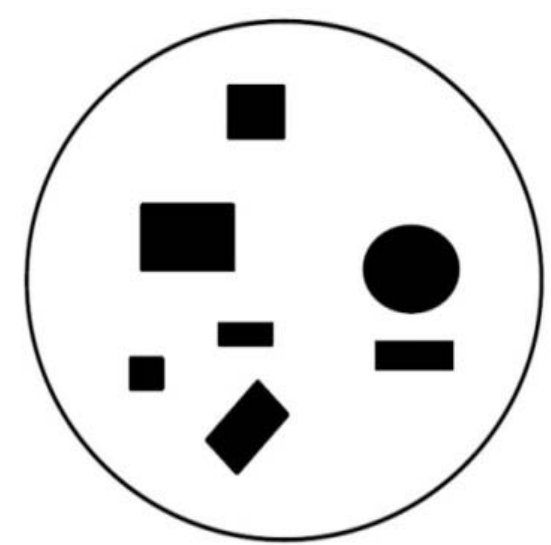

(b)

Figure 1: (a) Map-1 with less obstacles (b) Map-2 with more obstacles

We have compared our results of our algorithm in the different environment. Figure 1(a) and 1(b) show the map with less obstacle and with more obstacles respectively. Figure 2(a) and 2(b) show the path planning with less obstacles and more obstacles respectively. Comparison between two different environments is given in Table 2.

Table 1 Some Parameter used in the simulation

\begin{tabular}{|l|l|}
\hline Map Size & $\mathbf{2 4 X 2 4}$ \\
\hline Robot Sensor Range & 8 cell units \\
\hline Team Size & 4 Robots \\
\hline Robots Initial position & $\begin{array}{l}\text { Intersection between } \\
\text { circumference } \\
\text { Outer circle and line } X=Y\end{array}$ \\
\hline
\end{tabular}




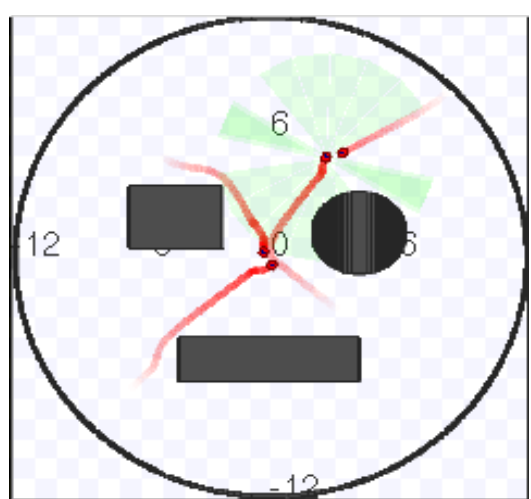

(a)

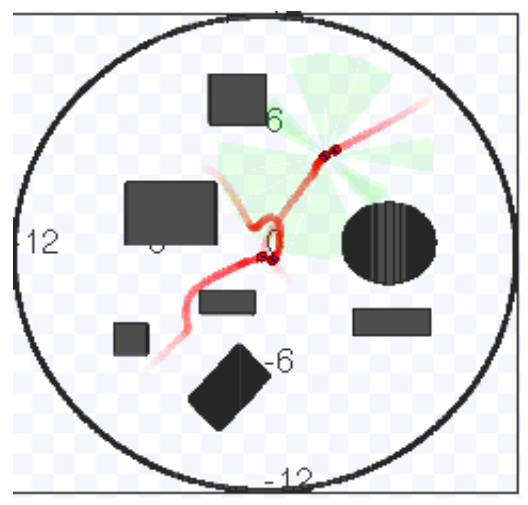

(b)

Figure 2: (a) Path planning with less obstacles (b) Path planning with more obstacles

Table 2 Comparison between different environments

\begin{tabular}{|l|l|l|}
\hline Environments & $\begin{array}{l}\text { Path } \\
\text { planning } \\
\text { without } \\
\text { failure }\end{array}$ & $\begin{array}{l}\text { Path } \\
\text { planning } \\
\text { with failure }\end{array}$ \\
\hline Environment 1 & 0 min $51 \mathrm{sec}$ & $1 \mathrm{~min} 04 \mathrm{sec}$ \\
\hline Environment 2 & $1 \mathrm{~min} 02 \mathrm{sec}$ & $1 \mathrm{~min} 33 \mathrm{sec}$ \\
\hline
\end{tabular}

From Figure 3 (a), cost Vs. local goals for environment 1, here, we have taken the reading of cost of each robot when they reach the local goal. Cost is in terms of time that means it is measured is second. We conclude that robot in the 1st sector has the minimum cost to reach the global goal. Hence, we can say that sector- 1 is the optimum sector to reach the goal with minimum cost. But there is the basic margin between robot to taking the cost to reach the global in the 1st sector and 3rd sector. Here, it is also clear that it is having less number of obstacles as compared to environment 2. From Figure 3(b), cost Vs. local goals for environment 2, here, we have taken the reading of cost of each robot when they reach local goal. We conclude that robot in the 3rd sector has the minimum cost to reach the global goal. Hence, we can say that sector-1 is the optimum sector to reach the goal with minimum cost.

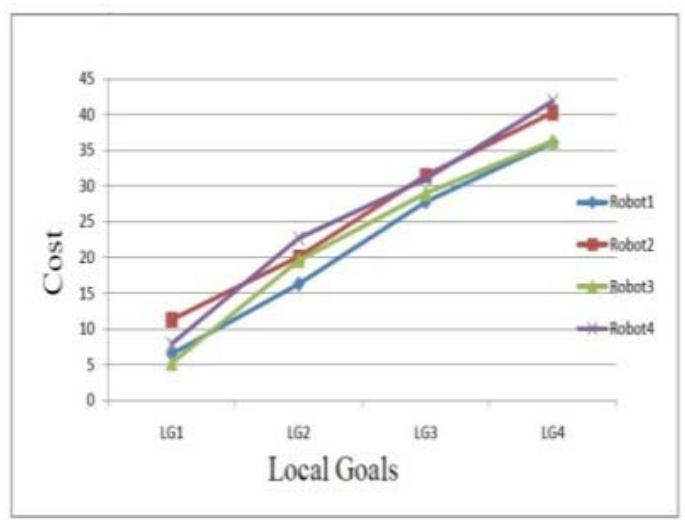

(a)

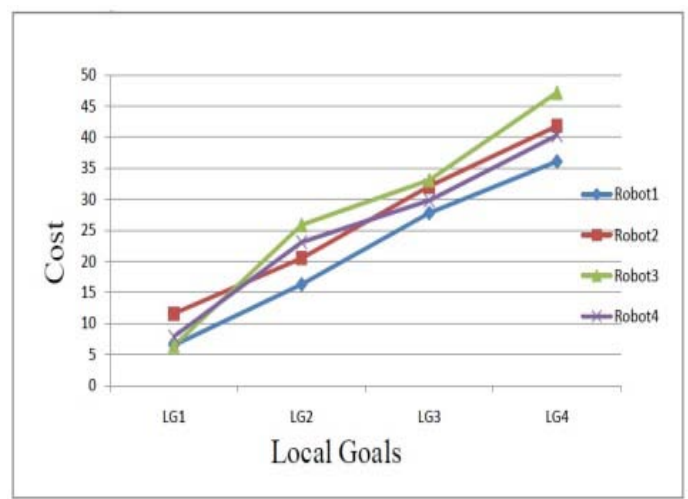

(b)

Figure 3 Cost Vs Local goals for environment (a) environment 1 (b) environment 2

\section{CONCLUSIONS}

We have proposed an efficient algorithm for the multirobot path planning to detect the fault if some of the robots are failed during the path planning. Our main concern is to find the shortest path without collision as early as possible. Energy conservation always an important play role when we are doing the path planning and area exploration by using the multiple robots. Computer simulation presented that the team of robot complete their path planning and find the shortest path with or without any fault and this process take less energy consumption by using our proposed algorithm. This method is restricted to requires a synchronization between robots because every time it checks that anyone robot is failed or not via sending the message.

In this paper, we use stationary obstacles in the given environment. But in the near future, we can implement our proposed algorithm for the moving obstacles in the environment and in that situation fault can also detect if anyone robot will fail.

\section{REFERENCES}

[1] Upma Jain, R. Tiwari, S. Majumdar, S. Sharma ( 2012 ) , "Multi Robot Area Exploration Using Circle Partitioning Method", International Symposium on Robotics and Intelligent Sensors (IRIS ), Procedia Engineering 41, pp. 383 - 387. 
[2] Noam Hazon, Gal A. Kaminka(2008), “On redundancy, efficiency, and robustness in coverage for multiple robots", Journals of Robotics and Autonomous Systems, Vol-56, No-12, pp. $1102-1114$.

[3] D. Puig , M.A. Garcia, L. Wua(2011), “A new global optimization strategy for coordinated multi-robot exploration: Development and comparative evaluation”, Journals of Robotics and Autonomous Systems, Vol-59, No-9,pp. 635 - 653.

[4] S. Sariel, T. Balch(2006), "Efficient bids on task allocation for multi-robot exploration”, Proc. 19th International FLAIRS Conference.

[5] K.S. Senthilkumar, K.K. Bharadwaj(2012), "Multi-robot exploration and terrain coverage in an unknown environment", Journals of Robotics and Autonomous Systems, Vol-60, No1,pp. $123-132$.

[6] Weihua Sheng, Qingyan Yang, Song Ci, Ning Xi(2004), "Multi-robot Area Exploration with Limited-range Communications”, Proc. IEEE/RSJ International Conference on Intelligent Robots and Systems, Vol-2, pp. 1414 - 1419.

[7] Jenhui Chen, Li-Ren Li(2005), "Path Planning Protocol for Collaborative Multi-Robot Systems”, Proc. IEEE International Symposium on Computational Intelligence in Robotics and Automation, pp. $721-726$.

[8] Weihua Sheng, Qingyan Yang, Jindong Tan, Ning Xi(2006), "Distributed multi-robot coordination in area exploration", Journals of Robotics and Autonomous Systems, Vol-54, No12,pp $945-955$.

[9] DING Fu-guang, JIAO Peng, BIAN Xin-qian, WANG Hongjian(2005), "AUV Local Path Planning Based on Virtual Potential Field", Proc. IEEE International Conference on Mechatronics \& Automation, Vol-4, pp. 1711-1716.

[10] Ralf Regele, Paul Levi(2006), "Cooperative Multi-Robot Path Planning by Heuristic Priority Adjustment”, Proc. IEEE/RSJ International Conference on Intelligent Robots and Systems, pp. 5954-5959.
[11] Kian Hsiang Low, Geoffrey J. Gordon, John M. Dolan, Pradeep Khosla (2007), “Adaptive Sampling for Multi-Robot Wide-Area Exploration", Proc. IEEE/RSJ International Conference on Robotics and Automation, pp $755-760$.

[12] Wurm, K. M., Stachniss, C. Burgard, W. (2008), “Coordinated Multi-Robot Exploration using a Segmentation of the Environment”, Proc. IEEE/RSJ International Conference on Intelligent Robots and Systems, pp. $1160-1165$

[13] Pal A, Tiwari R, Shukla A(2011), "Multi Robot Exploration Using Modified A* Algorithm', Intelligent information and Data Base systems", Lecture Notes in Computer Science, Vol-6591 pp.506 - 516.

[14] Pal A, Tiwari R, Shukla A(2012), "Multi Robot Exploration Through Purning Frontiers", Journals of advanced materials research ,Vol 462, pp. 609-616.

[15] A. Solanas, M. A. Garcia(2004), "Coordinated multi-robot exploration through unsupervised clustering of unknown space", Proc. Int. Conf. on Intelligent Robots and Systems, Vol-1,pp. 717-721.

[16] Ling Wu et al. (2010), "Balanced Multi-Robot Exploration through a Global Optimization Strategy”, Journal of Physical Agents, Vol-4, pp. 35-44.

[17] Praneel Chand, Dale A. Carnegie(2014), “Towards a robust feedback system for coordinating a hierarchical multi-robot system”, Journals of Robotics and Autonomous Systems, Vol62, No-2, pp. $91-107$

[18] M. Younis, F. Senturk, K .Akkaya, S. Lee, F. Senel(2014), "Topology management techniques for tolerating node failures in wireless sensor networks: A survey”, Journals of Computer Networks, Vol-58, pp. $254-283$

[19] Masehian, E. Sedighizadeh, D.(2010), “A multi-objective PSO-based algorithm for robot path planning”, International Conference on Industrial Technology(ICIT), pp. $557-561$ 